\title{
High Grade Atrioventricular Block in Von Recklinghausen Disease: A Rare Phenomenon
}

\author{
Glenmore Lasam ${ }^{\mathrm{a}, \mathrm{c}}$, Marcelina Lasam ${ }^{\mathrm{b}}$
}

\begin{abstract}
A case of a 73-year-old woman with a history of von Recklinghausen disease (neurofibromatosis type 1) who presented initially with a gradual onset of shortness of breath and lightheadedness with no associated fever, chills, angina, palpitations, cough, weight loss, night sweats, nausea, vomiting, or constipation. She was found to be severely bradycardic and in third degree atrioventricular block by her primary care physician. She was admitted in the hospital because of intermittent bouts of lightheadedness and progression of the shortness of breath. Twelve-lead electrocardiogram documented the high grade atrioventricular block. Chest radiograph showed subtle mild pulmonary congestion. Transthoracic echocardiogram revealed mild concentric hypertrophy and normal systolic function with no regional wall motion abnormalities or evidence of significant valvular disease. Pacemaker was inserted and her symptoms improved significantly.
\end{abstract}

Keywords: Third degree atrioventricular block; Neurofibromatosis type 1; Von Recklinghausen disease; Pacemaker; Lightheadedness

\section{Introduction}

High grade atrioventricular block in von Recklinghausen disease (neurofibromatosis type 1) is a very rare occurrence. An immediate pacemaker insertion is essential to abolish the progression of heart block symptoms and to avoid deleterious complications associated with it.

\section{Case Report}

A 73-year-old woman with history of von Recklinghausen disease (neurofibromatosis type 1) presented to the hospital because of shortness of breath and lightheadedness which

Manuscript submitted December 29, 2017, accepted February 28, 2018

${ }^{a}$ Morristown Medical Center, Morristown, NJ 07960, USA

bOverlook Medical Center, Summit, NJ 07901, USA

${ }^{\mathrm{c} C}$ Corresponding Author: Glenmore Lasam, Morristown Medical Center, Morristown, NJ 07960, USA. Email: glenmore_md@yahoo.com

doi: https://doi.org/10.14740/cr673w has been ongoing for 4 weeks. Before the occurrence of her complaints, she has been able to ambulate, climb stairs, and exercise in the gym without any problems. She had no prior documented cardiac illness and had a noncontributory family profile. She had no fever, chills, angina, palpitations, cough, weight loss, nausea, vomiting, or constipation. She denied any travel, camping, or visit to the woods as well as any note of a tick bite. She claimed that she had gradual onset of shortness of breath and noticed bouts of lightheadedness which made her unable to perform her routine activities of daily living. The episodes of lightheadedness were brief but no frank syncope was noted. She consulted her primary care physician and was found to be severely bradycardic and in complete heart block seen on office electrocardiogram. She was sent to the hospital and upon arrival, she had an episode of lightheadedness associated with a run of wide complex tachycardia at the rate of about 270 beats per minute which terminated by itself.

She was not on distress and has an adequate oxygenation. Vital signs were within normal range except for the bradycardia on the 30s. She was alert, oriented, and coherent. Cardiovascular examination showed no carotid bruit, irregular heart rhythm, cardiac murmurs, or abnormal lung sounds appreciated. Also, she had warm extremities without clubbing, cyanosis, or edema. Comprehensive metabolic panel, hemogram, lipid panel, and glycosylated hemoglobin were within normal. Other tests which include antinuclear antibody, thyroid function test, and lyme antibodies were negative as well. Twelvelead electrocardiogram showed third degree atrioventricular block (Fig. 1). Transthoracic echocardiogram revealed mild concentric hypertrophy and normal systolic function with no regional wall motion abnormalities or evidence of significant valvular disease (Fig. 2). Chest radiograph demonstrated subtle mild pulmonary congestion (Fig. 3, left panel).

She was transferred to the coronary care unit in which a transvenous pacemaker was inserted and eventually switched to a permanent one (Fig. 3, right panel). Bedside telemetry documented appropriate capture of her pacemaker (Fig. 4). Her lightheadedness, tiredness, and dyspnea gradually improved thereafter.

\section{Discussion}

Neurofibromatosis type 1 (NF1) or von Recklinghausen disease is the most common type of the neurofibromatoses [1]. NF 1 is genetically transmitted through autosomal dominant 


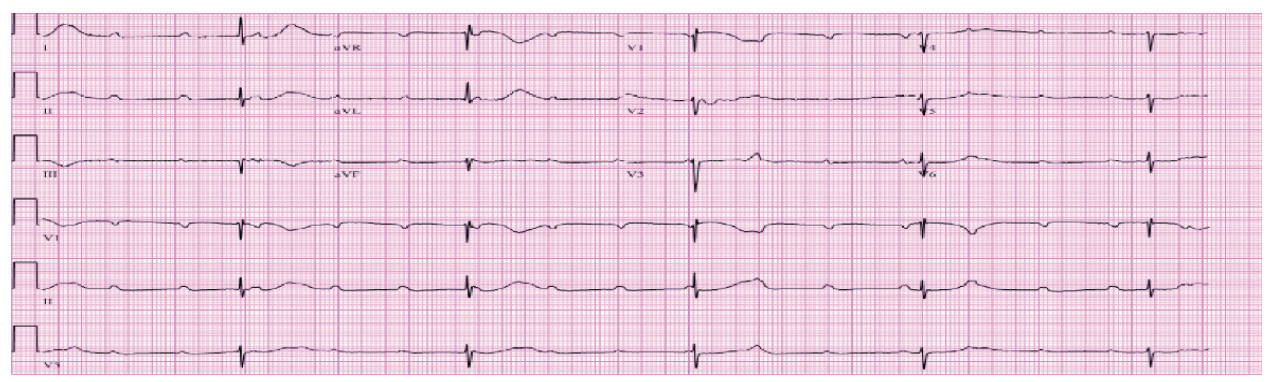

Figure 1. Patient's twelve-lead electrocardiogram revealing third degree atrioventricular block.

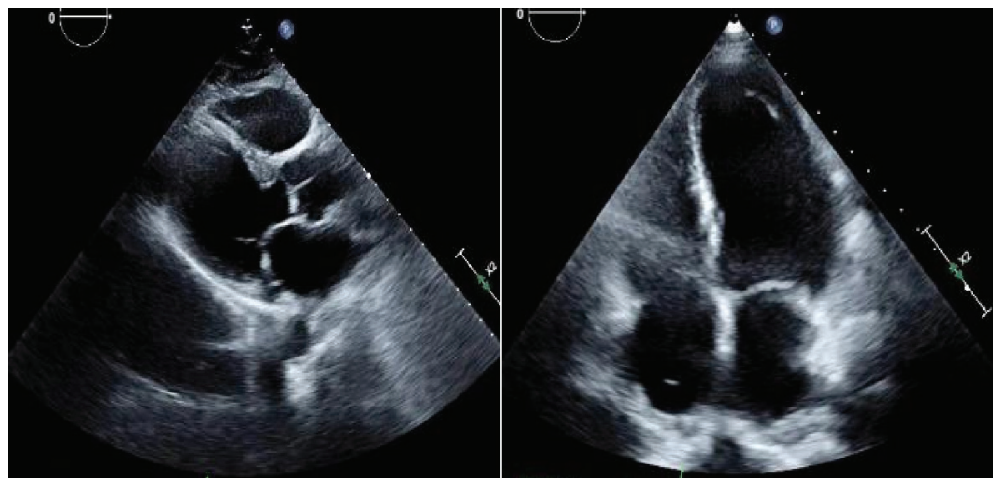

Figure 2. Parasternal view (left panel) and four chamber view (right panel) transthoracic echocardiogram still images revealing normal left ventricular cavity size with mild concentric hypertrophy. Also, no evidence of cardiac masses was detected.

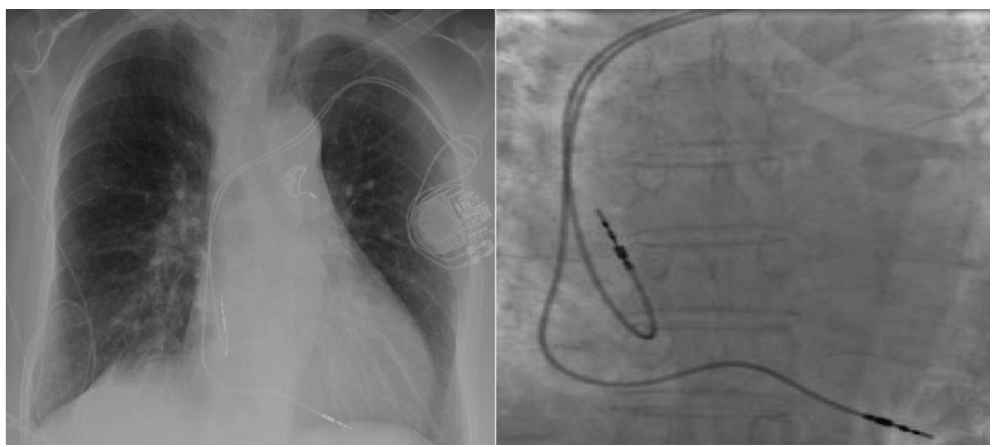

Figure 3. Chest radiograph demonstrating subtle mild pulmonary congestion (left panel). Dual chamber pacemaker leads showing insertion in the right atrium and right ventricular trabeculae (right panel).

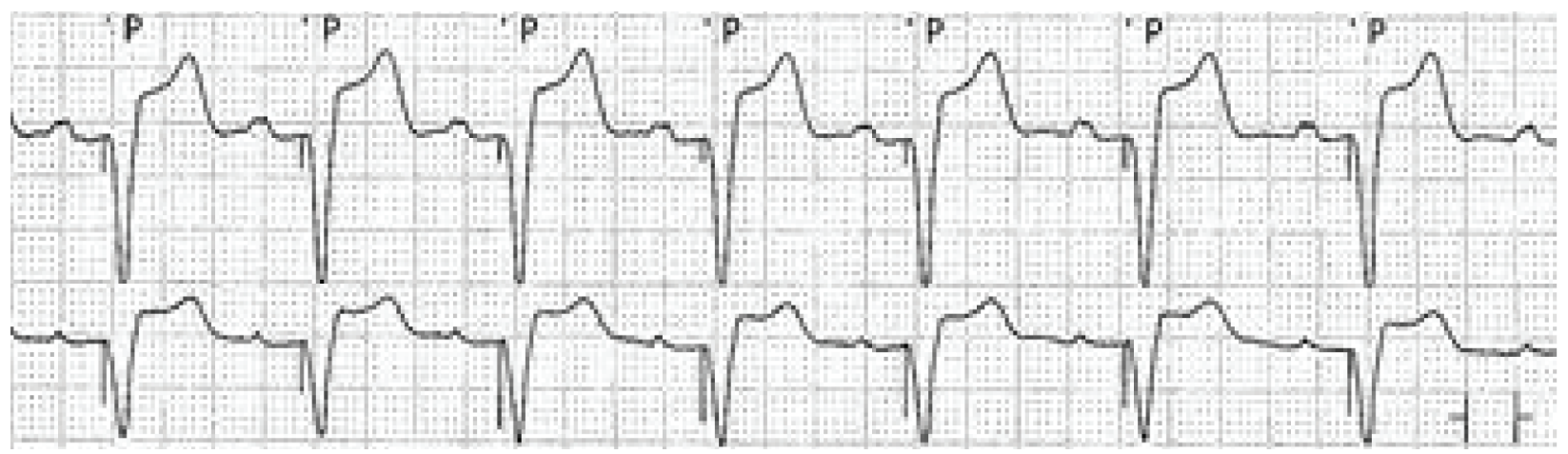

Figure 4. Patient's telemetry strip showing a ventricular paced rhythm after pacemaker insertion. 
pattern with an incidence of 1:2600 - 3000 individuals [2]. The inheritance is via familial pattern in half of the cases while the remainder was through sporadic mutations [3]. NF1 is caused by mutation in the $N F 1$ gene. $N F 1$ gene encodes neurofibromin which acts as a negative regulator of the Ras signal proteins [4]. The mutation causes deficiency in neurofibromin which does not accomplish the performance as a suppressor protein resulting into permanent transmission of the Ras signal [5].

NF1 has a diagnostic criteria developed by the United States National Institutes of Health (NIH) Consensus Conference in 1987 with an update in 1997 which had been commonly utilized for standard clinical care. The distinctive features of NF1 are multiple "cafe au lait" macules, axillary and inguinal freckling, neurofibromas, and iris hamartomas. The literature has been scarce on the association of neurofibromatosis and cardiovascular disease. Neurofibromatosis affects the heart rarely and the manifestations are less frequently recognized. In NF patients, renal artery stenosis and secondary hypertension had been elucidated in children [6] while hypertension secondary to pheochromocytoma had been described in adults [7].

In a large study of patients with definite NF1, cardiovascular malformations had been reported in $2.3 \%$ of patients which includes pulmonic stenosis (1\%), peripheral vascular abnormality $(0.85 \%)$, aortic coarctation $(0.21 \%)$, Watson or NF1Noonan syndrome $(0.17 \%)$, and other cardiac aberrations that comprises mitral valve prolapse, intracardiac tumor, murmur, and electrocardiogram abnormalities (1\%) [8]. Apparently, in a relatively remote and very small study that utilized a Holter monitor in NF1 patients, arrhythmias were found in $76 \%$ of patients which includes bradycardia, ventricular tachycardia, ventricular extrasystoles, supraventricular tachycardia, and supraventricular extrasystoles [9]. Also, a case of NF with evident manifestations of biventricular failure and atrial fibrillation had been elucidated and was discovered to have a benign neurofibroma extending to the interatrial septum [10].

There has been no reported evolution of third degree atrioventricular block in an adult patient with a NF1 and to our knowledge; our vignette is the first in the literature. However, a solitary case of NF1 in a 5-year-old child who developed sinus bradycardia with second-degree atrioventricular block that advanced at teenage years to a complete heart block had been documented [11]. Neurofibromas may be evident all over the body including the heart, thus, may contribute to the evolution of arrhythmias in NF1 patients.

A clinical vignette had been described on an isolated case of NF patient that developed a recurrent syncopal seizures attributed to sinus node dysfunction with complete resolution of symptoms after a permanent pacemaker implantation [12]. An isolated cardiac histological findings in a case of malignant peripheral nerve sheath tumor which originated from neurofibroma that metastasized to the heart revealed involvement of the myocardium and the common bundle of His, inferred to have contributed to the development of complete atrioventricular block [13].

To elucidate further the associated cardiac involvement in neurofibromatosis, supplementary cases need to be reported. Clinicians should be mindful with the varied features of neurofibromatosis as well as the involvement of other organs in order to develop a proper treatment approach and prevent detrimental outcomes.

\section{Conclusions}

Expeditious pacemaker insertion is imperative in patients with third degree atrioventricular block associated with neurofibromatosis. Prompt intervention is necessary in order to avert the detrimental complications of high grade atrioventricular block.

\section{Conflict of Interest}

The authors declare that they have no conflict of interest.

\section{References}

1. Korf BR. Neurofibromatosis type 1 (NF1): Pathogenesis, clinical features, and diagnosis. UpToDate, Waltham, MA. (Accessed on December 27, 2017).

2. Lammert M, Friedman JM, Kluwe L, Mautner VF. Prevalence of neurofibromatosis 1 in German children at elementary school enrollment. Arch Dermatol. 2005;141(1):71-74.

3. Evans DG, Howard E, Giblin C, Clancy T, Spencer $\mathrm{H}$, Huson SM, Lalloo F. Birth incidence and prevalence of tumor-prone syndromes: estimates from a UK family genetic register service. Am J Med Genet A. 2010;152A(2):327-332.

4. Buteica E, Stoicescu I, Burada F, Stanoiu B. Genetic and clinical considerations in six cases with neurofibromatosis type 1. Rom J Morphol Embryol. 2007;48(3):243248.

5. Koivunen J, Kuorilehto T, Kaisto T, Peltonen S, Peltonen J. Ultrastructural localization of NF1 tumor suppressor protein in human skin. Arch Dermatol Res. 2002;293(12):646-649.

6. Halpern M, Currarino G. Vascular lesions causing hypertension in neurofibromatosis. N Engl J Med. 1965;273:248-252.

7. Glushien AS, Mansuy MM, Littman DS. Pheochromocytoma; its relationship to the neurocutaneous syndromes. Am J Med. 1953;14(3):318-327.

8. Lin AE, Birch PH, Korf BR, Tenconi R, Niimura M, Poyhonen M, Armfield Uhas K, et al. Cardiovascular malformations and other cardiovascular abnormalities in neurofibromatosis 1. Am J Med Genet. 2000;95(2):108-117.

9. Malmcrona R, Zoller M, Rembeck B. Heart rhythm in patients with neurofibromatosis type 1. Cardiology. 1996;87(4):300-302.

10. Alaeddini J, Frater RW, Shirani J. Cardiac involvement in neurofibromatosis. Tex Heart Inst J. 2000;27(2):218-219.

11. Neiman HL, Mena E, Holt JF, Stern AM, Perry BL. Neurofibromatosis and congenital heart disease. Am J Roentgenol Radium Ther Nucl Med. 1974;122(1):146-149.

12. Verma S, Ahmed S, Gudapati SB, King DW, Mea- 
dor KJ, Ahmed R. Arrhythmias in neurofibromatosis. A case report and review of the literature. Cardiology. 2001;95(3):167-169.

13. Kitamura M, Wada N, Nagata S, Iizuka N, Jin YF, To- moeda M, Yuki M, et al. Malignant peripheral nerve sheath tumor associated with neurofibromatosis type 1, with metastasis to the heart: a case report. Diagn Pathol. 2010;5:2. 